\title{
DYNAMICS OF THE GALACTIC CENTER MOLECULAR CLOUDS
}

\author{
C. W. LEE
}

Harvard-Smithsonian Center for Astrophysics

60 Garden Street, $M S$ 42, MA 02138, USA

cwlee@cfa.harvard.edu

\author{
AND \\ H. M. LEE, H.B. ANN \& K.H. KWON \\ Department of Earth Science, Pusan National University \\ Pusan 609-735, Korea.
}

\begin{abstract}
We have performed Smoothed Particle Hydrodynamics (SPH) simulations in order to understand the dynamical structures of Galactic Center molecular clouds. In our study it was found that the structures of GC molecular clouds could be induced by the combined effects of a gravitational perturbation by rotating bar potential and the hydrodynamic collisions between the clouds.
\end{abstract}

\section{Introduction}

Rotating bar could provide significant non-axisymmetric perturbation to the orbits of molecular clouds in the disk. Non-circular velocity components develop as a result of such perturbations (Bally et al. 1988). The cloudcloud collisions will become inevitable in such circumstances. We performed SPH simulations to understand the general behavior of the gaseous disk under the influence of rotating bar and thereby the observed dynamics of molecular clouds in the GC.

\section{SPH Calculation}

In our SPH calculation (see Lee $e t$ al. (1998) for detail), the Galaxy is assumed to have four components: three axisymmetric components (massive halo expressed with the logarithmic potential, exponential disk and a 
compact bulge represented by a Plummer model) and one infinitely thin non-axisymmetric bar component.

Initially, SPH particles are uniformly distributed in a very thin axisymmetric disk. The initial velocity field of the SPH particles is generated such that these move on circular orbits compatible with the assumed potential distribution.

Most parameters are determined by the observed ones of the Galaxy, but the axis ratio of the bar is very uncertain. So, we considered several models with different values for bar's axial ratio. In this paper, we report the results of 4:1 axial ratio model. In all cases, we have fixed the angular speed of the rotating bar at $2.731 \mathrm{~km} \mathrm{~s}^{-1} \mathrm{kpc}^{-1}$ (Gehard 1996). This choice places the Corotation Radius (CR) at $2.7 \mathrm{kpc}$, the Inner Lindblad Resonance (ILR) at $1.2 \mathrm{kpc}$, and the Outer Lindblad Radius (OLR) at $6 \mathrm{kpc}$.

\section{Results \& Discussions}

Fig. 1-(a) shows the $l-v$ plot of SPH particles after several revolution. This can be compared with the CO $l-v$ map (Fig. 1-(b)) by Bitran (1987). The theoretical plot successfully reproduces the gas depletion region at $l \approx \pm\left(2^{\circ} \sim 4^{\circ}\right)$, but not the envelope (parallelogram structure) of highly velocity components between $l \approx 2^{\circ} \sim 2^{\circ}$. Such an envelope was interpreted as the presence of the $\mathrm{x} 1$ family of orbits by Binney et al. (1991). The orbit calculations of our model also indicate that the envelope can be produces by the $\mathrm{x} 1$ orbits (Lee et al. 1998). Therefore the fact that the SPH simulations do not show the high velocity envelope means that $\mathrm{x} 1$ orbits can be easily destroyed.

We have made another set of simulation that includes both SPH and collisionless particles. In the SPH simulations, almost all SPH particles are expected to experience hydrodynamic collisions because the resolution length is quite large. In our supplementary simulations with mixture of SPH and collisionless particles, the collisionless particles could be interpreted as those which have not undergone hydrodynamic collisions and therefore represent the less dense clouds. The collisionless particles are perturbed by the rotating bar but remains to have large velocity dispersion because there is no clear way to dissipate the orbital energy. The collisionless particles with large dispersion of the velocity can reproduce the high velocity envelope as well as forbidden velocity components (including Bania Clump 1) with $\mathrm{V}_{L S R} \approx 100 \mathrm{~km} \mathrm{~s}^{-1}$ at $l \approx-1^{\circ} \sim-6^{\circ}$ seen in CO map (Fig. 1-(d)). The fact that the HCN $l-v$ map resembles that of SPH particles while CO $l-v$ map is more like that of collisionless particles may indicate that the dense clouds traced by HCN molecules are formed by the hydrodynamic collisions between $\mathrm{CO}$ clouds. 

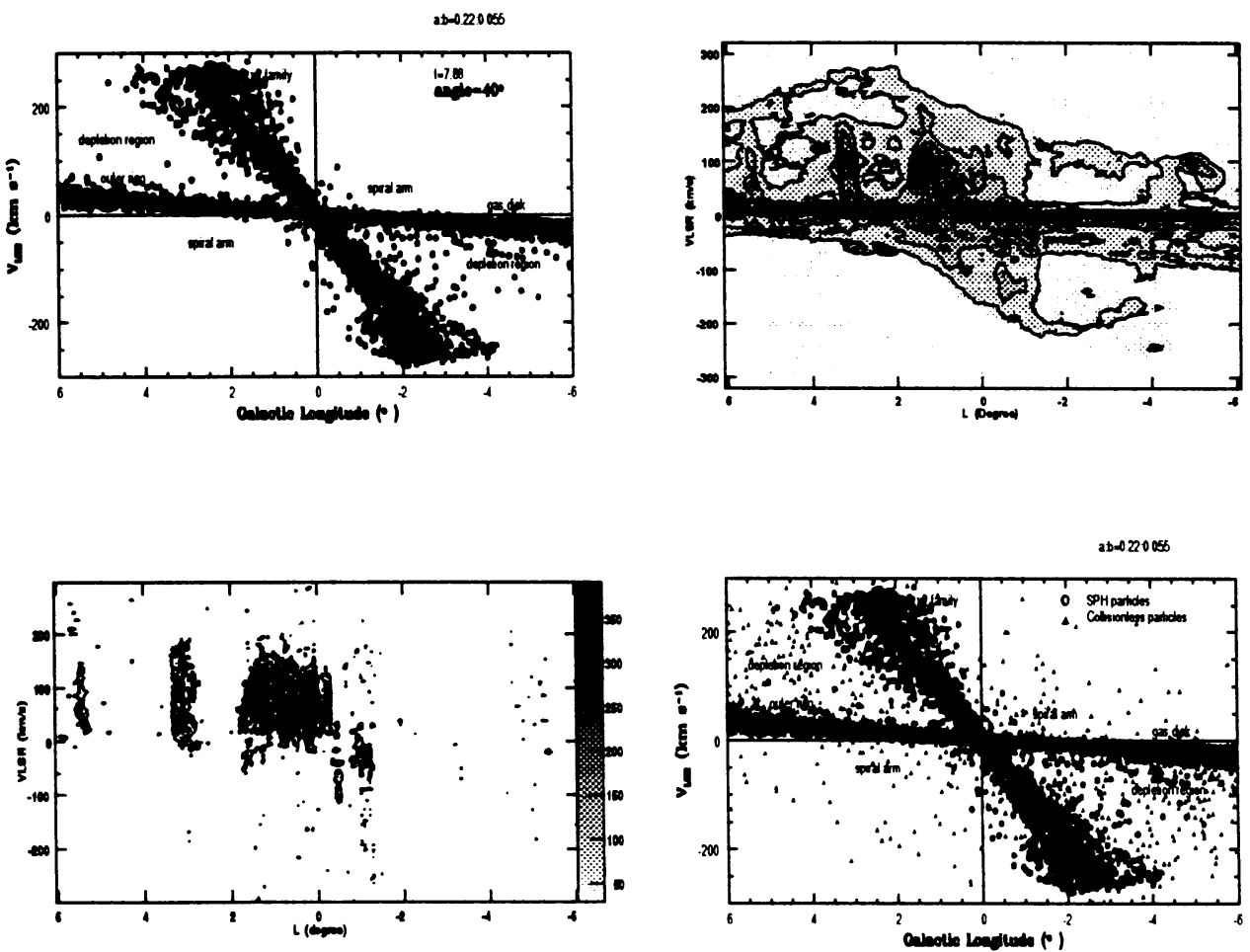

Figure 1. (a) The theoretical $l-v$ map of SPH particles at $t=7.88$ of B4-1 model (upper left). The viewing angle is $40^{\circ}$ from the direction of the major axis of the bar. (b) The Observational CO (1-0) $l-v$ map (Bitran 1987) (upper right). (c) The Observational HCN (1-0) $l-v$ map (Lee 1996) (lower left). (d) The theoretical $l-v$ map of a run of the model (B4-1) of the mixture of collisional (60\%) and collisionless particles (40\%) at the viewing angle of $40^{\circ}$ at $t=7.88$ (lower right).

The authors would like to acknowledge the financial support from SERI Supercomputer Center through CRAY R\&D Grant Program.

\section{References}

Bally, J., Stark, A. A., Wilson, R. W., \& Henkel, C. 1988, ApJ, 324, 223.

Binney, J., Gehard, O.E., Stark, A.A., Bally, J., \& Uchida K.I. 1991, MNRAS, 252, 210. Blitz, L., Binney, J., Lo, K. Y., Bally, J., \& Ho. P.T.P. 1993, Nature, $361,417$.

Bitran 1987, Ph. D. thesis, Univ. of Florida.

Gehard, O. E. 1995, in: Unsolved Problems of the Milky Way, IAU symp. 169, ed. L. Blitz, in press.

Lee, C.W. 1996, ApJS, 105, 129.

Lee, C.W. Lee, H.M., Ann, H.B., \& Kwon, K.H. 1998, in preparation. 